\title{
Child labor and childhood behavioral and mental health problems in Ethiopia
}

\author{
Atalay Alem ${ }^{1}$, Ababi Zergaw ${ }^{2}$, Derege Kebede ${ }^{2}$, Mesfin Araya $^{1}$, Menelik Desta ${ }^{4}$, Teferea Muche ${ }^{4}$, Debela Chali ${ }^{5}$, \\ Girmay Medhin ${ }^{6}$
}

\begin{abstract}
Background: According to ILO estimates, at least 180 million children aged 5 to 14 years are currently engaged in fulltime work in the developing countries. However, very little information exists about childhood behavioral and mental disorders in Ethiopia.

Objective: The objectives of this study are to estimate the prevalence and describe the nature of behavioral and mental health problems, as well as child abuse, nutritional problems, gross physical illness and injury among child laborers aged 8 to 15 years in Ethiopia. However, only the behavioral and mental health problems of the study population are examined here.

Method: A cross-sectional survey of children aged 8 to 15 years, and who were engaged in fulltime work in different formal and informal sectors non-laborers, was conducted in four major towns of Ethiopia. The screening instrument known as Reported Questionnaire on Children (RQC) and a diagnostic instrument known as the Diagnostic Interview for Children and Adolescents ( DICA) were used to detect symptoms and signs of behavioral and mental problems in the children.

Results: A total of 2000 child laborers and 400 non-laborers were interviewed using RQC to screen for probable cases of behavioral and mental problems. Of these, $50 \%$ of the laborers and $42 \%$ of the non-laborers were males. The mean age of the laborers was $13.8 \pm 1.8$ years while that of the non-laborers was $12.2 \pm 2.1$ years. More females (76.8\%) were found to have been engaged in domestic labor than males. The RQC interview screened $9.4 \%(n=226)$ of the children as probable cases of mental/ behavioral disorders, (14.0\% non-laborers and $8.5 \%$ laborers). The second stage DICA interview gave an overall prevalence of 5.5\% (4.9\% in laborers and $8.8 \%$ in non-laborers).

Conclusion: The prevalence of childhood behavioral and mental disorders in this study is within the range reported in previews studies conducted on children of the same age group. However, the lower prevalence of childhood disorders in the child laborers compared to that of the non-laborers found in the current study is probably due to selection bias or healthy workers effect. Thus, further study is recommended to explain this unexpected finding. [Ethiop.J.Health Dev. 2000;20(2):119-126]
\end{abstract}

\section{Introduction}

Engagement in economic activities at an early age and participation especially in hazardous and exploitative work could have a devastating effect on children's physical and mental development and might also cause irreversible damage leading to permanent disability (1). Children engaged in such activities are deprived of their childhood and potential by the demands of long hours and exposure to physical, social or psychological stress (2-4). Inadequate pay, high responsibility and lack of access to education all contribute to undermining the dignity and self-esteem of children. Apart from being detrimental to the full social and cognitive development of children, child labor is also frequently a cause of physical and emotional abuse (2-4). A study done in Nairobi, Kenya, indicated that out of 500 domestic servants between the ages of 6 and 15 years, $90 \%$ suffered from severe emotional distress, had symptoms of withdrawal and regression, were prematurely aging, as well as experiencing depression and low self esteem (5). Most of these children had sleep problems; exhibited phobic reactions to their employers, wetted their beds and, in some cases, exhibited behaviour not consistent with their chronological age.

Another WHO - sponsored review of studies on children working on the streets also identified the following conditions: distaste for regular, structured activities; excessive fatigue; heavy consumption of cigarettes and alcohol; infection with sexually transmitted diseases; defiance of parental control; recruitment into criminal activities, body deformity and stunting $(6,7)$.

Child prostitution is perhaps the worst form of child labor and has terrible consequences resulting from physical, emotional and sexual abuse. Prostitution may also result in sexually transmitted diseases including HIV infection, unwanted pregnancy and abortion. Such abuses lead to loss of self-esteem, physical and emotional illness, infertility, behavioral problems, substance abuse and even death (2) .

The exploitative nature of child labor was clearly shown by a study conducted in Colombia where domestic

\footnotetext{
${ }^{1}$ Department of Psychiatry, Faculty of Medicine, Addis Ababa University; ${ }^{2}$ Department of Community Health Faculty of Medicine, Addis Ababa University; ${ }^{3} \mathrm{WHO}$, Afro Regional Office, Brazzaville; ${ }^{4}$ Amanuel Specialized Mental Hospital, Addis Ababa; ${ }^{5}$ Department of Pediatrics, Faculty of Medicine, Addis Ababa University; ${ }^{6}$ Aklilu Lemma Institute of Pathobiology, Addis Ababa University
} 
servants under 15 years of age were found to be working 50 hours a week on average (8).

ILO surveys have shown that there are at least 180 million children aged 5 to 14 years engaged in fulltime work in the developing countries (2). Surveys conducted in Brazil, Colombia, Ghana, India, Indonesia, Senegal and Turkey have also shown that, on average, $25 \%$ of children in that age group were engaged in some form of economic activity. That survey found that for one-third of them it was a principal activity and for the remaining two-third, it was a secondary activity (8).

In Ethiopia, as in most developing countries, children are forced to engage in economic activities from an early age (9). From the few studies conducted so far, there is good indication that the problem exists and child domestic labor and prostitution appear to be ever increasing problems among female children in Ethiopia $(10,11)$. Child laborers both in rural and urban areas carry out unpaid work at home or in family enterprises (12). In urban areas large numbers of female child laborers are engaged in domestic labor while in rural areas both male and female child laborers work in agricultural fields.

Estimates of the prevalence of psychiatric disorders among children, identified using instruments, Reporting Questionnaire for Children (RQC), Childhood Behavioral and Psychological Questionnaire (CBPQ) and psychiatric interview range between 5.2 and $25 \%(13,14)$.

One of the most recent studies conducted in Butajira, southern Ethiopia, using the Diagnostic Interview for Children and Adolescents (DICA) showed that 3.5\% of the children had at least one or more diagnoses of childhood behavioral and emotional disorders (15).

However, well-designed studies that depict the relationship between psychosocial stressors faced by children and their emotional and other health problems do not exist. One ILO sponsored Pilot Study done on 1000 children (528 laborers and 472 non-laborers) in Addis Ababa; using DICA gave a prevalence of $20.1 \%$ and $12.5 \%$ for one or more psychiatric disorders among child laborers and non-laborers, respectively (16).

There is little information about the socio-economic activities and related health problems of working children in Ethiopia and, as a result, child labor has remained hidden. This study was thus conducted with the objective of depicting the relationship between child labor and childhood behavioural and mental, as well as physical and nutritional problems. The study was conducted in four major cities of the country by the Department of Psychiatry, Faculty of Medicine, Addis Ababa University, in collaboration with Amanuel Specialized Mental Hospital. This paper addresses only behavioral and mental problems in relation to child labor. The study involved children between 8 and 15 years of age engaged in economic activity whether they were paid or unpaid.

\section{Methods}

Study area: This study was conducted in Addis Ababa, and three other regional towns of Ethiopia, - Nazareth, Awassa and Bahirdar. These study sites are relatively more industrialized cities in the country which is one of the factors leading people, including small children, to migrate from rural to urban areas with the hope that they will have better employment opportunity and thereby be economically better off than in the rural areas. The cities are also thought to represent populations with some degree of socio-economic and cultural diversity.

Addis Ababa has more than two million inhabitants and serves as the political and economic capital of the country. Nazareth, located $100 \mathrm{Kms}$. east of Addis Ababa has 127,842 dwellers and is the seat of the Government of Oromia Regional State. Oromia is the largest region in Ethiopia in terms of geographic area and population size. Roads leading to the eastern regions of the country as well as to the ports of Djibouti and Assab pass through the town. Thus, it serves as one of the economic gates of the country. Awassa and Bahirdar Towns are located 273 and $565 \mathrm{Kms}$. south and north of Addis Ababa, respectively. Awassa is the seat for the government of the Southern Nations, Nationalities and Peoples Regional State while Bahirdar is the seat of government of Amhara Regional State. Awassa is a cash crop harvesting area and the main road to southern Ethiopia and Kenya passes through this town. It has a population of 69,169 while Bahirdar is a very rapidly growing town with a population size of 96, 140. The population in Addis Ababa city and the three regional states constitute nearly $85 \%$ of the country's population (9).

Study design and population: A cross sectional survey of child laborers and non-laborers was made in these urban areas to estimate the prevalence of mental, physical and nutritional problems associated with child labor and compare the result with that of non- laborers. The study population constituted working children who were engaged in domestic work, weaving, street work, commercial sex work and those working in establishments (shops, garages, hotels, carpentries, and metal workshops). The age range was restricted from 8 to 15 because in a previous study done in Addis Ababa, it was observed that children below this age had difficulty understanding the concepts in the survey questionnaires (16).

Sampling: Two different approaches, systematic and purposive sampling, were used to select the different groups of child laborers for the study. Before the actual survey was launched, a qualified public health professional collected background information about the number of hotels, restaurants, small scale industries and 
the projected population of each study site from the 1994 census to allow for a proper application of the sampling procedure. The lists of sub-districts (kebele) and that of the households within the respective city councils were used to calculate cumulative populations and the sampling interval (cumulative total population/number of clusters). Then five kebeles from each study site were selected using a probability proportional to size. After identifying an initial starting household using the random number method, subsequent households were visited until 20 domestic laborers were selected from each kebele. In a household where a child laborer was identified, one non-laborer child aged 8 to 15 years was randomly picked from the same household or from the neighboring household to represent the non-laborer group. To select child laborers in establishments and streets, the purposive sampling method was employed. Commercial areas were the main focus of this sampling and teenage commercial sex workers were picked from brothels.

By employing the purposive sampling method we collected the same sample size as the domestic laborers from other categories of child labor (commercial sex workers, street workers, workers in establishments and weavers). This procedure may violate the rules of assumption in sampling. However, instead of taking an arbitrary sample size from those target groups on the streets and establishments different from domestic laborers and non-laborers, we felt that taking the same sample size would give enough leverage.

Sample size: Based on the available local evidence (9, $12,15,17,18)$ we assumed a $12 \%$ prevalence of childhood behavioral and mental disorders, with a 95\% confidence interval, a $4 \%$ tolerance of sampling error, a design effect of 2 and a $20 \%$ allowance for non-response to calculate our sample size of child domestic laborers. The same sample size was used in each of the remaining category of child laborers (i.e, child laborers in the streets, establishments, child sex workers, weavers and non-laborers). This produced a total sample size of 2400 study subjects (2000 laborers and 400 non-laborers). This sample was equally distributed in the four study sites to include 100 subjects from each category of interest.

Instrument: Two questionnaires were used to detect the mental and behavioral problems of the children in a two stage design. In the first stage, the self Reporting Questionnaire for Children (RQC) was used to screen probable cases of behavioral and mental problems. In the second stage, the Diagnostic Interview for Children and Adolescents (DICA) was used for confirmatory diagnostic interviews on those screened positive by the RQC interview. In this particular study, sections of DICA that were meant to pick symptoms of certain emotional problems such as mood, anxiety, elimination and somatization disorders were used because these disorders had previously been shown to be more commonly detected problems in Ethiopian children in previous studies(15-17). This decision was taken by the investigators because of assumed resource limitation. $\mathrm{RQC}$ is a ten - item questionnaire developed by WHO for screening childhood mental and behavioral disorders(19). This questionnaire has been translated, back translated, validated and used in Ethiopia previously(20). DICA is a widely used instrument which was developed in Washington University, Seattle, USA, as a diagnostic instrument for children and adolescents (21). It is a highly structured instrument designed to be used by lay interviewers to detect childhood mental and behavioral disorders according to the Diagnostic Statistical Manual (DSM) classification of mental disorders. This instrument was also translated, tested and used in Ethiopia previously $(15,22)$.

Data collection: Data collection was conducted from October 2001 to May 2002 using those questionnaires. In each of the study sites data collection took two months. Data collectors in all sites were locally recruited and comprised of 10 high school graduates, two health assistants and two psychiatric nurses. The health assistants assessed physical signs of illness and made anthropometric measurements of the subjects while psychiatric nurses acted as supervisors. A one week training on the proper administration of the questionnaires was given to all of the research personnel. The training included both theoretical and practical sessions. The practical sessions involved role plays among the trainees and field practices. A short guideline on how to administer the questionnaires was prepared and given to the data collectors and supervisors for quick reference in the field.

Children who gave one or more positive responses to the RQC questions were interviewed using DICA to obtain a specific diagnosis. The health assistants performed physical and nutritional assessments of the study subjects using standardized instruments. Weight was measured using digital scales, height with a vertically fixed tape and the appropriate tools were also used for vital sign measurement. The supervisors monitored day-to-day data collection activities and checked the completeness of the questionnaires in the field. The research assistant closely monitored all aspects of the data collection.

Data processing: Data entry and analysis used the DICA program, SPSS version 10.0 and EPI-INFO version 6. Double data entry system was employed to minimize data entry error. Mean values for continuous variables and proportions for categorical variables were used as descriptive summary measures. A chi-square test was used to compare the magnitude of mental health problems among laborers and non laborers and to determine if there was a difference in socio-demographic 
characteristics between the two groups that may account for any difference in the prevalence of mental health problems, other than that of being a laborer or nonlaborer. In the next stage, a multiple logistic regression method was employed using forced entry, forward selection and backward selection methods to identify possible confounding socio-demographic factors. Any RQC diagnosis confirmed by the DICA interview was included as a dependent variable in the logistic model. The following factors were included as co-variates: sex, age group (4 levels), ethnicity (8 levels), religion (4 levels), and child labor (2 levels). Statistical significance was assumed whenever the p-value was less than $5 \%$.

Ethical Consideration: Ethical approval was obtained from the Ethical Committee of Amanuel Hospital and the National Ethical Clearance Committee. Informed consent was obtained from each respondent and the collected information was kept confidential. Confirmed cases of children with mental health problems were provided with treatment during the survey and connection with the nearby health services was facilitated for those requiring long term treatment.

\section{Results}

A total of 2000 child laborers and 400 non-child laborers aged 8 to 15 years were interviewed. Fifty percent of the child laborers were males and $42 \%$ of the non-child laborers were males. Around 58\% of the child laborers and $32.3 \%$ of the non-laborers were between 14 to 15 years of age. The mean age of the child laborers was $13.8 \pm 1.8$ years while that of the non-laborers was 12.2 \pm 2.1 years. The Amhara ethnic group was predominant (52.1\%) amongst child laborers followed by Gurage (12.5\%), Wolayta (11.2\%) and Oromo ethnic groups (10.5\%). The majority were Orthodox Christians (72.1\%), followed by Muslims (17.2\%) and Protestant Christians (10.1\%). The laborers and non-laborers were significantly different in their socio-demographic characteristics such as age, sex, ethnicity and religion. A significant proportion of the child laborers (78.5\%) were literate, the highest level of educational attainment being grade 8 (Table 1 ).

Eighty six percent of the laborers were brought up by their parents and 65\% of them had siblings aged less than 15 years. More female laborers (76.8\%) were engaged in domestic labor while more males were employed in other establishments.

The socio-demographic characteristics of the parents of the child laborers and non laborers differed in some ways (data not shown). The parents of the laborers were disadvantaged in terms of income, education and marital status (separation and divorce). About 3.0\% of the child laborers and $1.5 \%$ of the non-laborers had at least one parent with a history of mental illness. Over $11.0 \%$ of the child laborers smoked cigarettes while $1.8 \%$ and

Table 1: Socio demographic characteristics of child laborers and non-laborers, Ethiopia, 2004

\begin{tabular}{|c|c|c|c|}
\hline Characteristics & Laborers (\%) & Non-laborers (\%) & P-value \\
\hline \multicolumn{4}{|l|}{ Sex } \\
\hline Male & 1010 (50.5) & $168(42.0)$ & \\
\hline Female & 989 (49.5) & $232(58.0)$ & 0.002 \\
\hline \multicolumn{4}{|l|}{ Age } \\
\hline $8-9$ yrs & 75 (3.8) & 52 (13.0) & \\
\hline $10-11$ yrs & 232 (11.6) & $94(23.5)$ & 0.000 \\
\hline 12-13 yrs & 539 (27.0) & 125 (31.3) & \\
\hline $14-15$ yrs & 1152 (57.7) & $129(32.3)$ & \\
\hline \multicolumn{4}{|l|}{ Ethnicity } \\
\hline Amhara & 1041 (52.2) & $228(57.0)$ & \\
\hline Oromo & 209 (10.5) & 63 (15.8) & \\
\hline Tigre & 45 (2.3) & 18 (4.5) & \\
\hline Guraghe & $250(12.5)$ & $34(8.5)$ & 0.000 \\
\hline Hadia & $14(0.7)$ & $1(0.3)$ & \\
\hline Kembata & 29 (1.5) & $8(2.0)$ & \\
\hline Wolayta & $224(11.2)$ & $35(8.8)$ & \\
\hline Others & $184(9.2)$ & $13(3.3)$ & \\
\hline \multicolumn{4}{|l|}{ Religion } \\
\hline Orthodox & 1442 (72.2) & 313 (78.3) & \\
\hline Protestant & $202(10.1)$ & $42(10.5)$ & 0.006 \\
\hline Muslim & 343 (17.2) & 42 (10.5) & \\
\hline Others & $11(0.6)$ & $3(0.8)$ & \\
\hline \multicolumn{4}{|l|}{ Grade attained } \\
\hline No schooling & 363 (8.2) & $17(4.3)$ & \\
\hline $1-4$ & 792 (39.6) & $176(44.0)$ & \\
\hline $5-8$ & 778 (38.9) & $183(45.8)$ & 0.162 \\
\hline $9-12$ & $67(3.4)$ & $24(6.0)$ & \\
\hline Total & $2000(100.0)$ & $400(100.0)$ & \\
\hline
\end{tabular}

Sum of study subjects does not add up to total values in some sections because of missing values 
1.3\% used stimulants like cannabis and Shisha (a mixture that may include tobacco, honey, hashish and spices and is smoked from an oriental tobacco pipe), respectively; 5.3\% chewed khat and 8.3\% drank alcohol every day.

The aggregate prevalence of childhood behavioral and mental disorders according to the first stage RQC interview was $9.4 \%(n=226),(8.5 \%$ in child laborers and $14.0 \%$ in non-laborers, $\mathrm{p}<0.001$ ) (Table 2). When logistic regression analysis was employed where any RQC diagnosis confirmed by DICA interview was taken as dependent variable and all socio-demographic variables and child labor were entered as covariates, the difference of occurrence of mental health problems between the two groups remained statistically significant with about a two fold increased risk $(\mathrm{OR}=1.86,95 \% \mathrm{CI}=$ 1.24, 2.77) (Table 4). The result was not changed when forward and backward selection methods were used.

Table 2: Mental health problems (RQC diagnosis) among child laborers and non-laborers, Ethiopia, 2004

\begin{tabular}{lccc}
\hline Characteristics & Laborers (\%) & Non-laborers (\%) & p-value \\
\hline Any RQC diagnosis & $170(8.5)$ & $56(14.0)$ & $<0.001$ \\
Speech problem & $11(0.6)$ & $3(0.8)$ & 0.422 \\
Sleep Problem & $31(1.6)$ & $8(2.0)$ & 0.319 \\
Convulsion & $17(0.9)$ & $4(1.0)$ & 0.473 \\
Repeated headache & $75(3.8)$ & $21(5.3)$ & 0.108 \\
Disappear from home & $31(1.6)$ & $4(1.0)$ & 0.283 \\
Stealing & $18(0.9)$ & $7(1.8)$ & 0.108 \\
Excess fear & $18(0.9)$ & $12(3.0)$ & 0.002 \\
Retarded & $40(2.0)$ & $16(4.0)$ & 0.017 \\
Difficulty getting along with others & $16(0.8)$ & $1(0.3)$ & 0.196 \\
Elimination problems & $25(1.3)$ & $11(2.8)$ & 0.027 \\
Other mental health problems & $7(0.4)$ & $3(0.8)$ & 0.224 \\
\hline
\end{tabular}

Figures of RQC diagnosis items do not add up to the total any RQC diagnoses (226) because one respondent could have more than one diagnosis

With respect to specific mental health problems, a significant difference between child laborers and nonlaborers was observed for excessive fear $(p=0.002)$, retardation $(\mathrm{p}=0.017)$, and elimination problems $(p=0.027)$. When this analysis was restricted to a comparison between child domestic laborers and nonlaborers, there was no longer a significant difference in the prevalence of these disorders. However, domestic laborers reported difficulty in getting on with other people more often $(2.5 \%)$ than the non laborers $(0.3 \%)$ $(\mathrm{P}=0.006)$.

Out of the 226 RQC positive children 133 fulfilled DSM III-R diagnostic criteria for behavioral and mental disorders as measured with DICA. Hence, the overall prevalence of mental disorders in the study population was $5.5 \%$ which was $4.9 \%$ in the laborers and $8.8 \%$ in the non-laborers $(\mathrm{P}=0.002)$. A higher proportion of disorders was found in domestic child laborers compared to non-laborers and other categories of child laborers. The major emotional problems identified in child laborers were phobia (3.1\%), enuresis (1.0\%) and separation anxiety (0.4\%). Combinations of these disorders were also seen in some of the child laborers. Although the occurrence of each disorder was not high in both groups the proportions of phobia and enuresis with phobia were significantly higher among non laborers (Table 3).

Table 3: Mental disorders (DICA diagnosis) among child laborers and non-laborers, Ethiopia, 2004

\begin{tabular}{lcccc}
\hline Characteristics & Laborers & Non-laborers & Total (\%) & P-value \\
\hline Any DSM III-R Diagnosis & $98(4.9)$ & $35(8.8)$ & $133(5.5)$ & 0.002 \\
Separation anxiety & $7(0.4)$ & $2(0.5)$ & $9(0.4)$ & 0.651 \\
Phobia & $62(3.1)$ & $21(5.3)$ & $83(3.5)$ & 0.036 \\
Enuresis & $20(1.0)$ & $4(1.0)$ & $24(1.0)$ & 1.000 \\
Encopresis & $0(0.0)$ & $1(0.3)$ & $1(0.0)$ & 1.000 \\
Enuresis with Phobia & $3(0.2)$ & $5(1.3)$ & $8(0.3)$ & 0.005 \\
Separation anxiety with phobia & $4(0.2)$ & $2(0.5)$ & $6(0.3)$ & 0.263 \\
Separation anxiety and phobia and & $2(0.1$ & $0(0.0)$ & $2(0.0)$ & 1.000 \\
enuresis & & & & \\
\hline
\end{tabular}


Table 4: Logistic regression model for prediction of group status "any DSM III-R diagnosis vs no diagnosis" of 2400 child laborers and non laborers based on selected socio-demographic variables

\begin{tabular}{|c|c|c|c|c|c|c|}
\hline $\begin{array}{l}\text { Step-1 } \\
\text { Variables entered into the } \\
\text { equation }\end{array}$ & B & Wald** & P-value & Chi-square & p-value & $\begin{array}{l}\% \text { correctly } \\
\text { predicted }\end{array}$ \\
\hline Variables in the model & & & & 8.300 & 0.004 & $94.4 \%$ \\
\hline Constant & -2.963 & 817.836 & 0.000 & & & \\
\hline $\begin{array}{l}\text { Prescribed anti-psychotics/ } \\
\text { antidepressant }\end{array}$ & 0.618 & 9.084 & 0.003 & & & \\
\hline \multicolumn{7}{|l|}{$\begin{array}{l}\text { Variables not in the model } \\
\qquad \text { Sex }\end{array}$} \\
\hline Female & & 1.086 & 0.297 & & & \\
\hline \multicolumn{7}{|l|}{ Age } \\
\hline 10-11 years & & 0.766 & 0.381 & & & \\
\hline $12-13$ years & & 0.744 & 0.388 & & & \\
\hline $14-15$ years & & 0.116 & 0.733 & & & \\
\hline \multicolumn{7}{|l|}{ Ethnicity } \\
\hline Oromo & & 0.004 & 0.952 & & & \\
\hline Tigre & & 0.181 & 0.671 & & & \\
\hline Gurage & & 3.087 & 0.079 & & & \\
\hline Hadiya & & 0.825 & 0.364 & & & \\
\hline Kembata & & 1.792 & 0.181 & & & \\
\hline Wolayta & & 0.081 & 0.776 & & & \\
\hline Others & & 0.902 & 0.342 & & & \\
\hline \multicolumn{7}{|l|}{ Religion } \\
\hline Protestant & & 0.523 & 0.470 & & & \\
\hline Muslim & & 0.132 & 0.716 & & & \\
\hline Others & & 0.757 & 0.384 & & & \\
\hline
\end{tabular}

** Score statistic instead of Wald statistic for variables not in the model

\section{Discussion}

The prevalence of mental and behavioral disorders in children in the current study was found to be much lower than our previous findings in the Addis Ketema child labor study, although it is within the range of reported prevalence from other studies in Ethiopia. The first general population survey for childhood mental disorders in Ethiopia in the 1960s, showed that 3-4\% of children of up to nine years of age and 5-10\% of those aged 10-19 years, were suffering from psychiatric illnesses (23).

The prevalence of childhood disorders from previous studies using the RQC as the sole measure ranged from $17.7 \%$ to $24.0 \%(14,17)$. Using a screening instrument to estimate the prevalence of general psychological disturbances may give an overall impression of the status of a community with regard to problems of mental health. However, for an intervention plan, knowledge about specific disorders is essential. Diagnostic instruments such as DICA were developed to identify specific disorders. For this reason DICA was translated from English into Amharic and its feasibility, acceptability and reliability were tested in Ethiopia before being employed in the community surveys (22). Prior to the current study, DICA had been used in two previous studies in Ethiopia. One was conducted in the rural district of Butajira, and the other one in the Addis Ketema district in Addis Ababa. The prevalence of childhood disorders in Butajira was reported to be 3.5\% while in Addis Ketema the prevalence was $16.5 \%$. In the child laborers it was $20.1 \%$ and in the non-laborers it was $12.5 \%(15,16)$.

The prevalence of childhood mental and behavioral disorders identified in the current study which is lower than the Addis Ketema study may be attributed to two basic design differences. First, the age-group under the study differed in that it was 5 to 15 years of age in the previous study compared to 8 to 15 years in the current study. Secondly, a screening instrument was used in the current study. This means that there may have been false negatives at the first screening stage. The screening instrument had only ten questions and the expected responses were "yes" or "no". Such responses might give spurious results unless they are probed further to determine the level of understanding of the respondents when answering the questions. Difficulty with conceptual validity using a similar instrument with adult respondents has previously been reported in Ethiopia (24). On the other hand instruments like DICA many help to probe questions to verify the validity of yes or no responses. Interviewing a sub-sample of RQC negatives 
with DICA would have given a better understanding of the situation. Another possible explanation is that using the same interviewer to administer both RQC and DICA to the same respondent may have introduced systematic error. The lower prevalence of the disorders in child laborers compared to non-laborers is hard to explain. Because of the 'healthy-worker' effect child laborers may be selectively retained in the workforce while those with illnesses may leave that population and join the nonlaboring population. As a result of this, the point prevalence of mental and behavioural disorders will be lower in child laborers. Healthy and robust laborers are also likely to be picked by using the purposive sampling method. Another possible, but very unlikely explanation might be that child labor is protective against behavioural and mental disorders in children because it might give some motivation and a sense of achievement for children who are from economically disadvantaged families.

Disorders were more common in the domestic child laborers than in the non-laborers and other categories of laborers. This might be explained by the degree of control exerted by their employers and the lack of personal freedom. Additionally, domestic child laborers work much longer hours than other groups of child laborers. They also encounter various types of abuse more frequently than the other groups. Abuse was shown to be commoner in this group of laborers in the previous Addis Ketema study with sexual abuse, exclusively reported by this group. Private homes are closed environments that could expose domestic workers to be victims of exploitation as well as to physical, sexual and emotional abuse, all of which are well-known risk factors for emotional disorders. Considering the specific disorders identified, anxiety disorders and phobia in particular, were shown to be the commonest ones. This finding is consistent with previous studies where phobia was shown to be the commonest disorder followed by elimination disorders. Elimination disorder was also the second commonest problem identified in this study, but the overall prevalence was much lower than those reported previously. Again the age difference may explain the difference as all of the previous studies included younger children as well. It is known that elimination disorder is commoner in the younger age group.

In conclusion, the results of this study were not found to concur with our previous child labor study conducted in Ethiopia that showed a higher prevalence of childhood behavioural and mental disorders in child laborers compared to non laborers. This might be attributed to methodological differences between the two studies. Additional research is strongly recommended for a better understanding of the situation in this special group of the population.

\section{Acknowledgement}

The study was fully funded by the International Labor Organization. Amanuel Hospital is highly acknowledged for administering and disbursing the fund. We would also like to thank all the children, data collectors, supervisors and data entry clerks who took part in the study.

\section{References}

1. International Labour Organization: Child labourTargeting the intolerable (Report). Geneva, Switzerland 1998.

2. International Labour Organization: Report of the Director-General: A Future without Child Labour. Geneva, Switzerland 2002

3. Nangia P, Pinto RP. Situational analysis of children in especially difficult circumstances in Union Territory of Delhi-with focus on working and street children. New Delhi, India, 1988.

4. International Labour Organization: Eliminating the worst forms of child labour. A Practical Guide to ILO Convention No. 182, Handbook for Parliamentarians, 2002.

5. Onyango P, Kayongo-Male D. Child labor and health: Proceedings of the First National Workshop on Child Labor and Health in Kenya, University of Nairobi, Kenya 1983.

6. World Health Organization: Children at work, special health risks, WHO Study Group Technical Report. Geneva, Switzerland, 1987.

7. Bekele A, Myers W. First things first in child labour: Eliminating work detrimental to children (Report). Geneva, Switzerland. 1995.

8. Forastieri V: Children at work: Health and safety risks. 2nd edition 2002.

9. Central Statistical Authority: The 1994 Population and Housing Census of Ethiopia, Vol. II, Addis Ababa, Ethiopia. 1999.

10. Kifle A. Ethiopia child domestic workers in Addis Ababa: A rapid assessment, International Labour Organization and International Program on the Elimination of Child Labour. Geneva, Switzerland, 2002.

11. Ayalew T: Child prostitution in Bahar Dar town, Thesis Submitted to the School of Graduate Studies. Addis Ababa University, Addis Ababa, Ethiopia, 1999.

12. Central Statistical Authority: Statistical Report on the 1999 National Labour Force Survey. Addis Ababa, Ethiopia, 1999.

13. Giel R, van Luijk J: On the significance of broken home in Ethiopia and prevalence of psychiatric illness among children. Br J Psychiatry 1969; 114:957-961.

14. Mulatu MS: Prevalence and risk factors of psychopathology in Ethiopian children. J Am Acad Child Adolesc Psychiatry 1995;34:100-109. 
15. Ashenafi Y DK, Desta M, and Alem A: Mental and behavioural disorders in children in Butajira: I. Prevalence Estimates: The East African Medical Journal 2001;78:308-311.

16. Fekadu D, Alem A and Hagglot B. The prevalence of mental health problems in Ethiopian child laborers. Journal of Child psychology and psychiatry 2006. In press.

17. Tadesse B, Kebede D, Tegegne $T$, Alem A: Childhood behavioural disorders in Ambo District, western Ethiopia. I Prevalence estimate. Acta Psychiatr Scand 1999a;100:92-97.

18. International Labour Organization: Report on the National Workshop on Child Labour Policy and Action Program for Ethiopia. Addis Ababa, Ethiopia. 1996.

19. Giel R, Dearango MV, Climent, CE. et al.: Childhood mental disorders in primary health care: Results of observations in four developing countries. Paediatrics 1981;68:677-683.
20. Tadesse B, Kebede D, Tegegne $T$, Alem A: Childhood behavioural disorders in Ambo District, western Ethiopia. II. Validation of the RQC. Acta Psychiatr Scand 1999b;100:98-101.

21. Herjanic B, Reich W: Development of a structured psychiatric interview for children: Agreement between child and parent on individual symptoms. J Abnorm Child Psychol 1982;10:307-324.

22. Kebede M, Kebede D, Desta M, Alem A: Evaluation of reliability, acceptability \& feasibility of the Amharic version of DICA. Ethiop J Health Dev 2000;14:13-21.

23. Giel R, Bishaw M, Van Luijk J: Behaviour disorders in Ethiopian Children. Psychiat Neurol Neurochir 1969;72:395-400.

24. Kortmann F: Problems of practising psychiatry in Ethiopia. Ethiop Med J 1988;26:77-83. 